\title{
Immersive human-machine interface for controlling the operation of the Telerescuer robot
}

\author{
Krzysztof A. Cyran, Wojciech Moczulski, Dariusz Myszor, Marcin Paszkuta, Adam Rurański, \\ Mateusz Kalisch, Józef P. Cyran, Marek Adamczyk, Anna Timofiejczuk
}

\begin{abstract}
The paper presents the modes of operation of Telerescuer robot controlled by an innovative human-machine interface. The robot is designed to perform inspections during the rescue actions in subterranean areas of the coal mines affected by catastrophic events. In order to make navigation of the robot as simple as possible, the immersive human-machine interface has been developed, whose purpose is not only to navigate, but also to supervise semi-autonomous mission of the robot. In addition, the paper presents experiments with triangulation of the point clouds resulting from the 3D scanner installed on the robot as well as architecture of other main components of the whole Telerescuer system, developed in collaboration of academic and industrial partners from Poland, Czech Republic and Spain.
\end{abstract}

Keywords-human-machine interaction, inspection robot, rescue action, teleimmersion.

\section{Introduction}

Telerescuer is a system composed of a mobile robot and an immersive human-machine interface by which an operator is supervising the whole mission. The mission of Telerescuer robot is to inspect hardly accessible subterranean areas of coal mines affected by catastrophes [1]. This is rapidly growing area of application of unmanned vehicles. The comprehensive overview of the state-of-the-art platforms used for inspection in coal mines is presented in [2]. Telerescuer system's originality lies in innovative approach used for supervising the mission of the robot, which may operate autonomously or be remotely controlled by an operator using various modes of the immersive human-machine interface. The purpose of this interface is to support an operator with the impression of being remotely present at distant place, where robot is localized. This is achieved by knowledge based virtual teleportation technology developed in the project by SkyTech Research company. The prototype of the robot has been constructed by Silesian University of Technology (the Leader European Telerescuer Consortium), the low-level control subsystem [3] has been developed by Vysoka Skola Banska from Czech Republic, and the communication channel has been designed by Universidad Carlos III de Madrid. A brief description of the Telerescuer system will be given in section 2, whereas section 3 will present the main features of immersive humanmachine interface and the modes used to supervise the robot mission.

Krzysztof.A. Cyran, Dariusz Myszor, Marcin Paszkuta

Institute of Informatics, Silesian University of Technology, Gliwice, Poland; Wojciech Moczulski, Marek Adamczyk, Anna Timofiejczuk

Institute of Fundamentals of Machinery Design, Silesian University of Techn., Gliwice, Poland;

Krzysztof.A. Cyran, Wojciech Moczulski, Dariusz Myszor, Marcin Paszkuta, Adam Rurański, Mateusz Kalisch, Józef P. Cyran

SkyTech Research, Gliwice, Poland.
In section 4 we present conclusions regarding the current state of the system and point out the plan for its final demonstration in operational environment.

\section{Telerescuer Description}

The overall look of Telerescuer robot is presented in Fig. 1. Its design has been done with the use of FEM analysis. Fig. 2 reports on the example of robot arm, how particular elements have been developed, starting from simplified 3D models, towards the final mature stage of the construction.

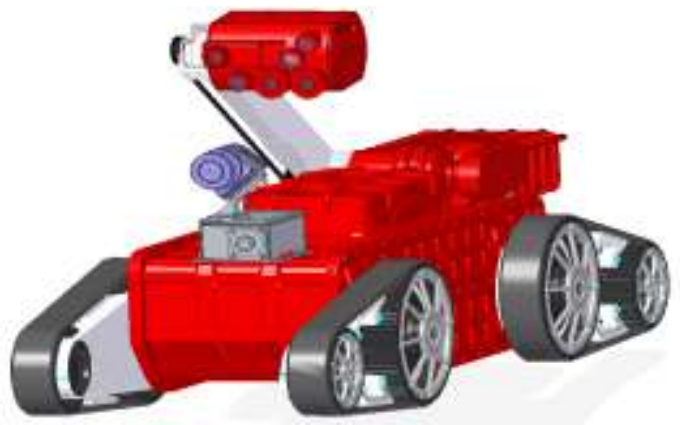

Fig. 1. Telerescuer robot. Source: Telerescuer project report.

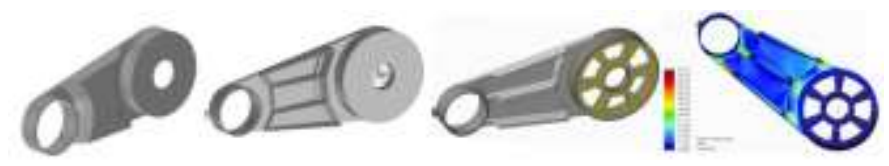

Fig. 2 Evolution of the arm during design process based on FEM analysis. Source: Telerescuer project report.

At the top of the robot, there is an arm for methane sensor and a cylinder in which the cameras are installed. The construction of the arm is given in Fig. 3. This is a complex element composed of flexible shaft, belt tensioners and belt drives as the methane sensor must be activated as high as possible. Only then, measuring of methane concentration will give reliable results, what is of a great importance form planning the rescue action.

The robot is also using a complex optical system. The cameras are placed in the cylinder, whose shape has been carefully designed as a compromise between its size and capacity for carrying the cameras. In order to provide to the operator the good quality picture of the subterranean area, the Consortium decided to use stereoscopic camera supporting immersive environment of the human-machne interface, FishEye camera supporting with wide angle view of the terrain, and the thermal camera supporting and operator with infra red imaging. The location of these cameras in the cylinder is given in Fig. 4. 


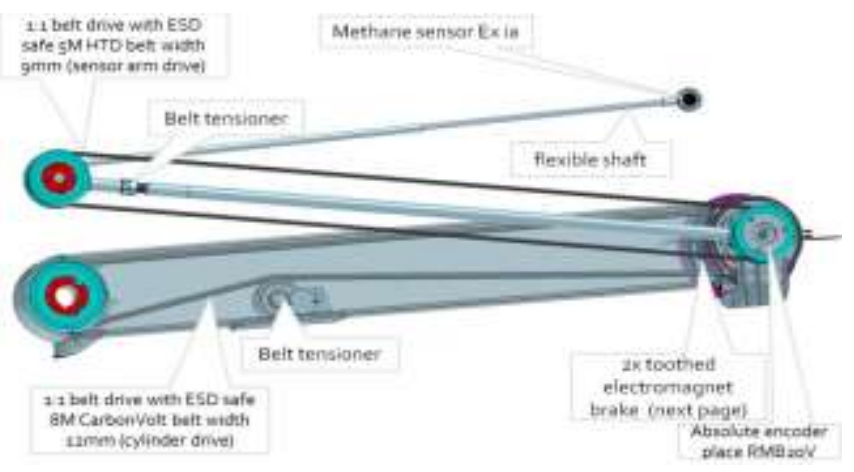

Fig. 3. Robot arm for positioning sensors at required for measurements position. Source: Telerescuer project report.

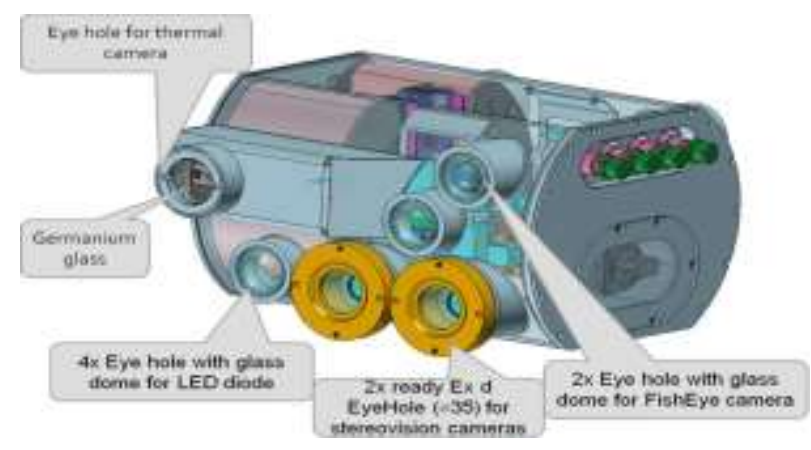

Fig. 4. The visual stereoscopic camera, infrared camera, and other sensors mounted in the cylinder of Telerescuer robot. Source: Telerescuer project report.

For transmission of the stereoscopic images, the broad band communication channel is designed. It uses a fiber optic as a transmission medium (see Fig. 5). Such system requires a spool with the optic fiber (see Fig. 6), which will be placed on the ground during the movement of the robot towards area of catastrophic event.

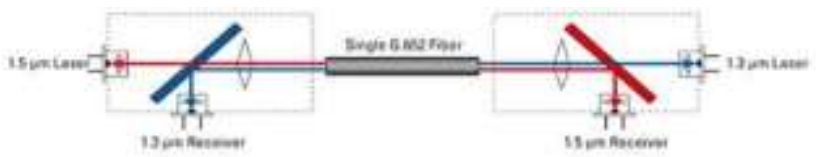

Fig. 5. Bidirectional communication subsystem using fiber optic. Source: Telerescuer project report.

During robot's return, the autonomous mode based on previously done 3D map building will be applied, and therefore, a broad-band communication using fiber optic will not be required at this stage. In order to build accurate 3D maps of the surrounding terrain, the 3D laser scanner (see Fig. 8) will be utilized during forward move of the robot.
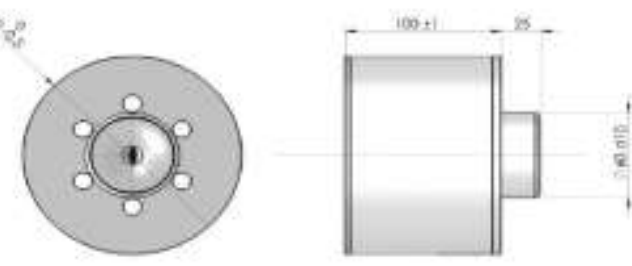

Fig. 6. Optical fiber spool mounted on the robot and used as brad band communication media in Telerescuer system. Source: Telerescuer project report.

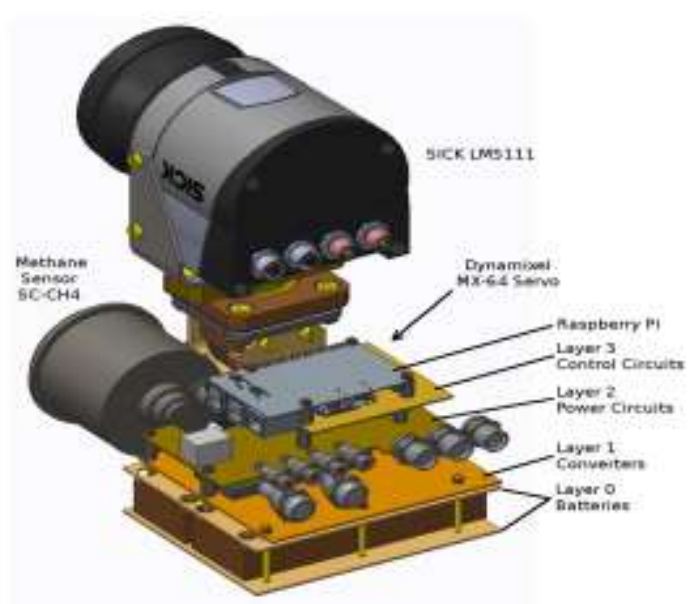

Fig. 8. 3D Laser scanner - spatial organization of internal electrical equipment. Source: Telerescuer project report.

The result of 3D scanning is a cloud of enormous number of points. To obtain from this cloud useful information, reduction of the data needs to be done. In our experiments done in the project, the cloud of points representing the surrounding subterranean area (see Fig. 9) has been transformed in a triangulation process to a set of triangles (see Fig. 10). However, the number of triangles after this operation, still remains too large, and therefore, the next step is smoothing. After performing this step, primarily detected by 3D scanner clouds of points surfaces are now represented by relatively small number of objects (see Fig. 11), which simplify internal representation of 3D maps required for autonomous operation during robot's return.

Autonomous movement subsystem requires position and orientation information for correct path finding. Localization information is also shown to operator by operator interface.Localization uses three different types of information: encoders data, AHRS (attitude and heading reference system), and point cloud matching using various 3D mapping models [4], [5]. The data from encoders inform about number of rotations that robot wheels have performed. Given that position between wheels is constant, it is possible to calculate path from a change in orientation and in position.

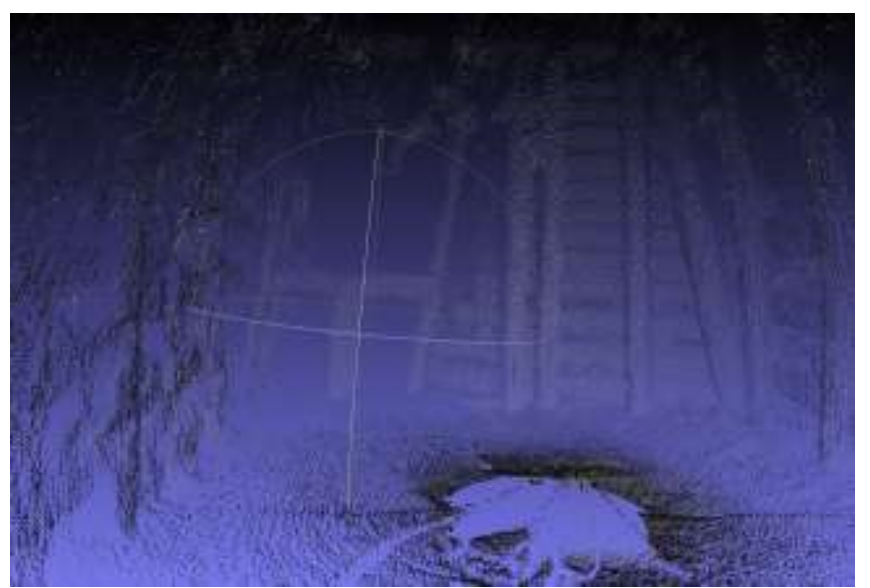

Fig. 9. Illustration of a cloud of points representing subterranean area. 


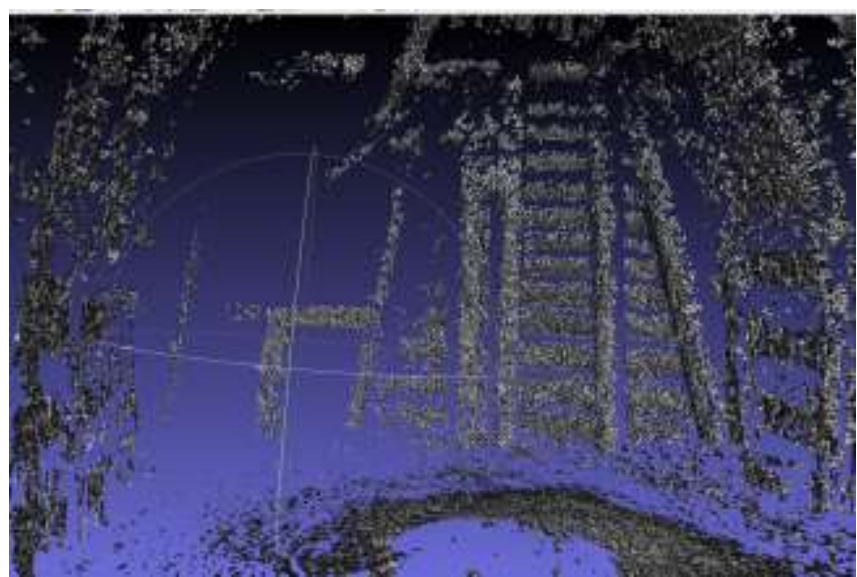

Fig. 10. Cloud of points transformed by SkyTech Research to a mesh of very small triangles using PCL (Point Cloud Library).

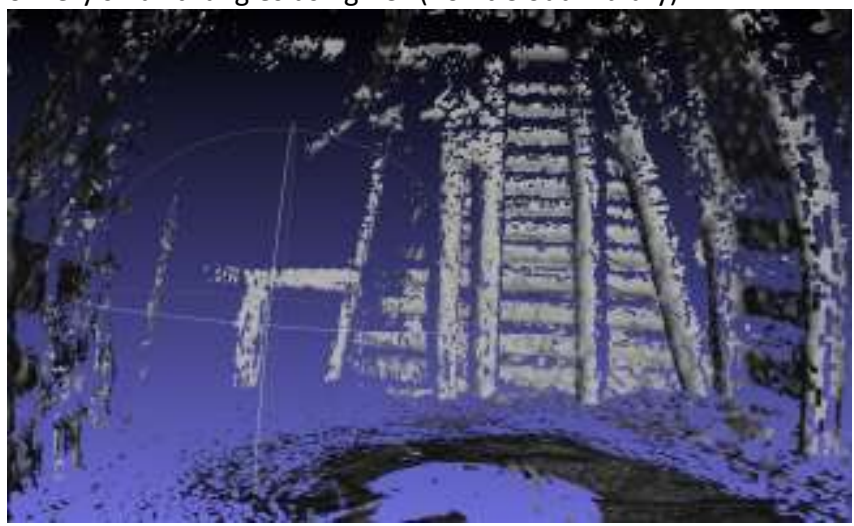

Fig. 11. Representation of subterranean area after triangulation has been smoothed by SkyTech Research.

The second source, commonly referred to as IMU (inertial measurement unit), provides information about current orientation and accelerations. This does not allow to correctly calculate distance as a standalone procedure, but can be used as a part in fusion process (see Fig. 12). The last source is point cloud matching. Even though scanner subsystem uses only rough estimation as its input data, it is still possible to get position corrections to improve localization after each scan. The localization subsystem does not use camera images, mostly due to assumed poor quality given environmental conditions (lack of light and similarity between frames/lack of characteristic points).

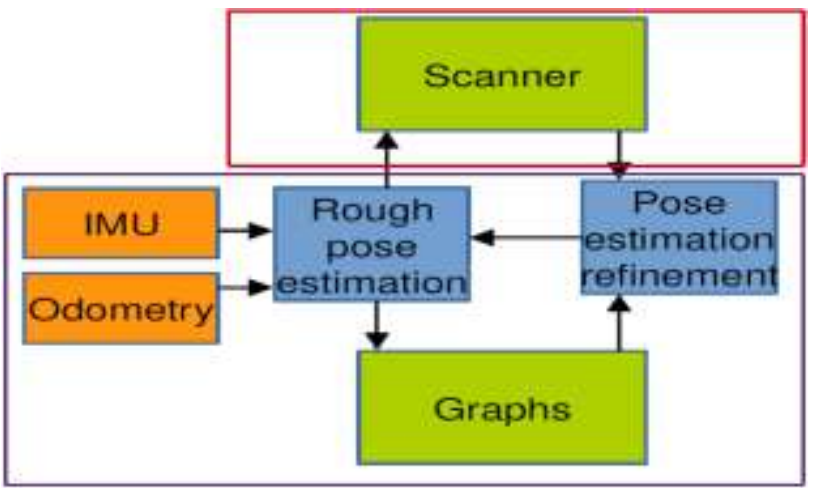

Fig. 12. Diagram of data fusion method applied in Telerescuer for localization. Source: Telerescuer project report.

\section{Immersive human-machine interface}

In many situations, the autonomous operation of the robot will not be possible and then supervision of the robot operation will require human intervention. In that case the operator will deactivate autonomy and select one of three modes of operation of the mobile robot (see Fig. 13) using immersive human-machine interface. This interface consists of two parts:

- hardware that is exploited for robot movements controlling and also serves as a platform for visualization of the data obtained from the robot;

- software components that are responsible for presentation of the data on provided hardware components.

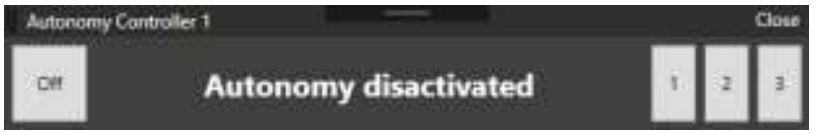

Fig. 13. Deactivation of autonomous mode. Source: Telerescuer project report.

TeleRescuer operator station software has been developed using Object Oriented Programming Principles, which define code metrics (see Table 1 for their values achieved by SkyTech Research).

TABLE I. TELERESCUER PROJECT METRICS OBTAINED BY CODE METRICS FUNCTIONALITY AVAILABLE IN VISUAL STUDIO 2015 IDE.

\begin{tabular}{|l|l|l|l|l|}
\hline $\begin{array}{l}\text { Maintainability } \\
\text { Index (avg) }\end{array}$ & $\begin{array}{l}\text { Cyclomatic } \\
\text { Complexity } \\
\text { (avg) }\end{array}$ & $\begin{array}{l}\text { Depth of } \\
\text { Inheritance } \\
\text { (avg) }\end{array}$ & $\begin{array}{l}\text { Class } \\
\text { Coupling } \\
\text { (avg) }\end{array}$ & $\begin{array}{l}\text { Lines of } \\
\text { Code in } \\
\text { the TR } \\
\text { project } \\
\text { (sum) }\end{array}$ \\
\hline $87 \%$ & 6 & 2.5 & 3.5 & 82418 \\
\hline
\end{tabular}

The definition of these metrics are as follows:

- Maintainability Index represents difficulty in maintenance of a project. Higher values indicate better maintainability. TeleRescuer project achieves high $87 \%$ of $100 \%$ score in this area,

Cyclomatic Complexity represents, ,amount of decision logic in source code function" [6]. E.g. simple function, without if statements can have complexity of 1-2, and each if statement increases this value by 1 . High value of cyclomatic complexity suggests that method is getting too complicated. Software developed for Telerescuer humanmachine interface is characterized by proper level of cyclomatic complexity, this level is caused by the type of algorithms utilized in the code.

Depth of Inheritance is defined as "the maximum length from the node to the root of the tree". Deeper position of a class in the hierarchy, the greater is the number of methods that it will inherit. As a result it is harder to predict its behavior. Additionally, design complexity increases, as more 
classes and methods are involved. This increases probability of errors in the code. On the other hand, higher depth of inheritance means that there is a greater potential for code reuse [7]. It is suggested that DIT around 5-6 should be the upper limit.

Class Coupling is a metric of how many classes a single class uses. Class coupling has been shown to be an accurate predictor of software failure and recent studies have shown that an upper-limit value of 9 is the most efficient [8]. Software for implementation of TeleRescuer human-machine interface project keeps good score at the level of 3.5.

Some of software components of this interface include:

Pad controller - a debug panel which supports analysis of the problem that might occur such as button malfunction. In addition, it can be used for controlling the robot movements, when other types of controllers are not available (Fig. 14).

Saitek Pro Flight Yoke - a debug panel that presents the state of the plane controller and can be used in order to test whether all the functions of the controller work correctly. It should be utilized before every mission in order to confirm that the plane controller is functioning properly and to detect malfunctions that can be caused by e.g. a transportation process (Fig. 15).

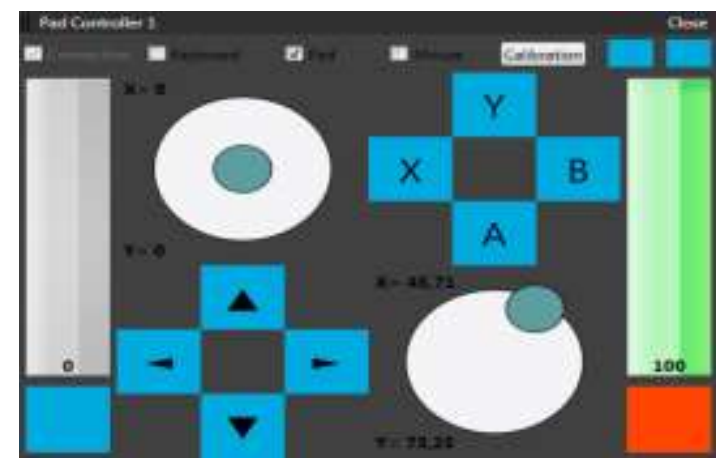

Fig. 14 Pad controller

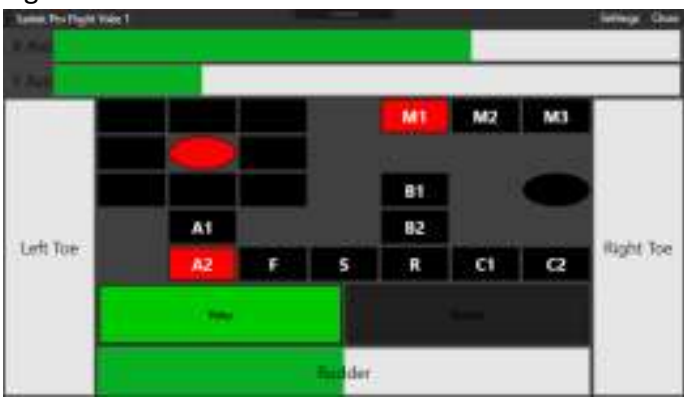

Fig. 15 Saitek Pro Flight Yoke

The software panel presented in Fig. 15, serves for debugging Saitek Pro Yoke, whose picture is given in Fig. 16.

The next important panel is that for presentation of $3 \mathrm{D}$ view of the subterranean area (see Fig. 17). The method for stereovision is based either on passive anaglyph approach (the cheapest) or other more advanced active approaches of 3D displaying. In addition, the head up display (HUD) control, located directly on the top of the window is developed. It introduces an innovative functionality of augmented reality into the operator station, which allows the virtual teleportation of the operator into the scene of operation of the Unmanned Vehicle (mobile robot). The location of the control and the type of displayed information facilitates orientation in the operational space of the robot by presenting robot position in relation to the ground (Fig. 18). In addition, the position of robot arms, are presented on a separate panel (see Fig. 19).1

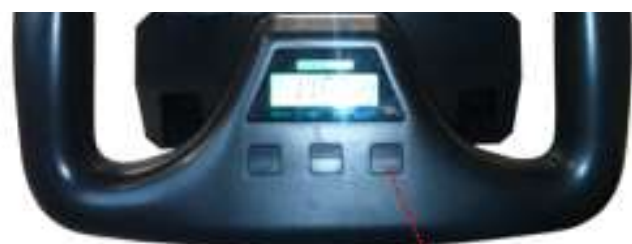

Fig. 16 Flight Yoke used in operator station to control robot.

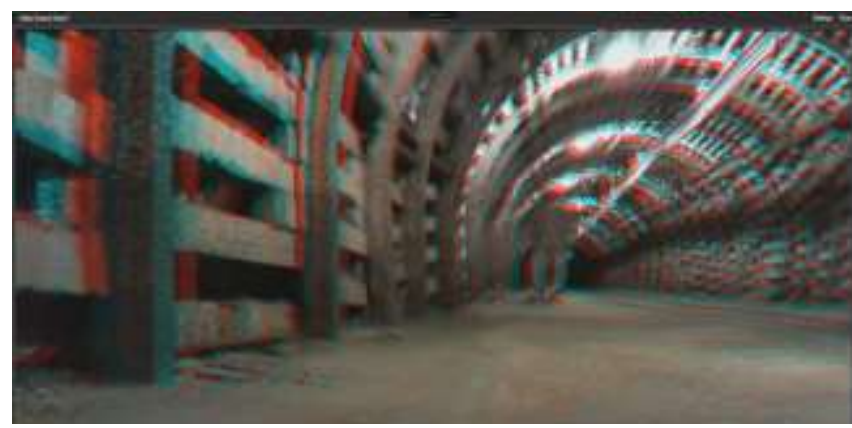

Fig. 17 Panel for 3D video.

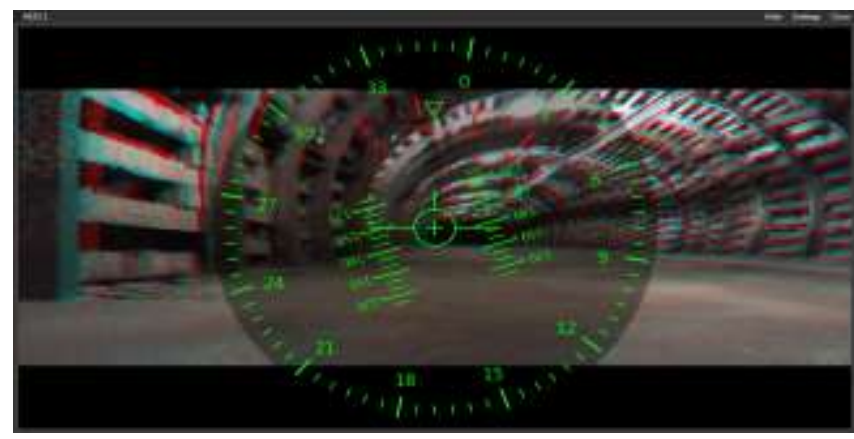

Fig. 18 HUD panel

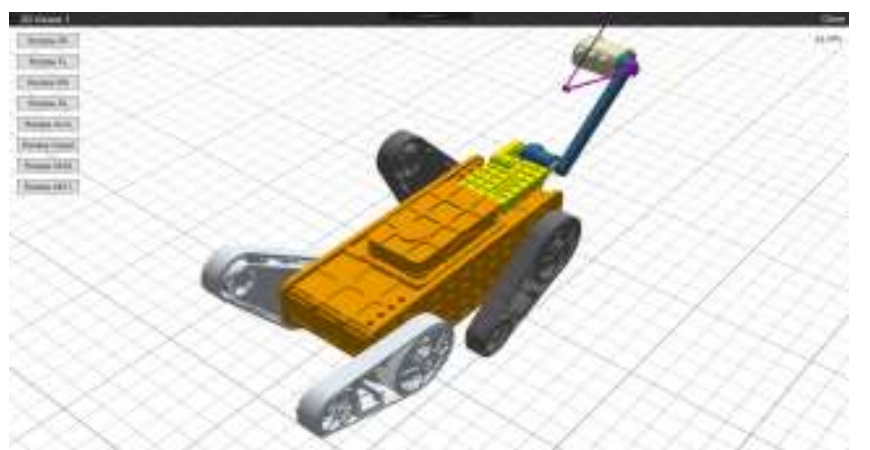

Fig. 19 3D robot view

The operator station communicate with the server using two different approaches, either "Command/Answer" or "Command/Auto answer". The first approach (Fig. 20) is used to set parameters on the server. When the server receives and correctly decodes the telegram, the server will send back the 
answer to the client. In most cases, an answer is only information for the client that the telegram has been received properly. Some of the commands use the Command/Answer approach to get some parameters or values from the server on demand.

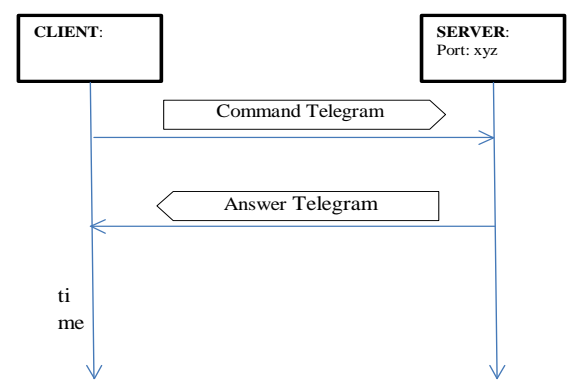

Fig. 20 Communication between the Client and the Server: Command/Answer approach

The second solution (Fig. 21) is used only for data received from the server. When the server receives a command from the operator station it starts to send back auto answer telegrams to the client. Answers are sent periodically and frequency of answers is set in the first command sent from the client to the server.

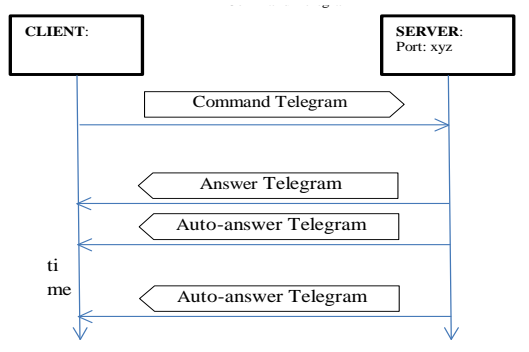

Fig. 21 Communication between Client and Server: Command/Auto answer approach

The design of the hardware and mechanical part of the operator station is presented in Fig. 22 and the visualization of the Telerescuer robot in operational environment is in Fig. 23.

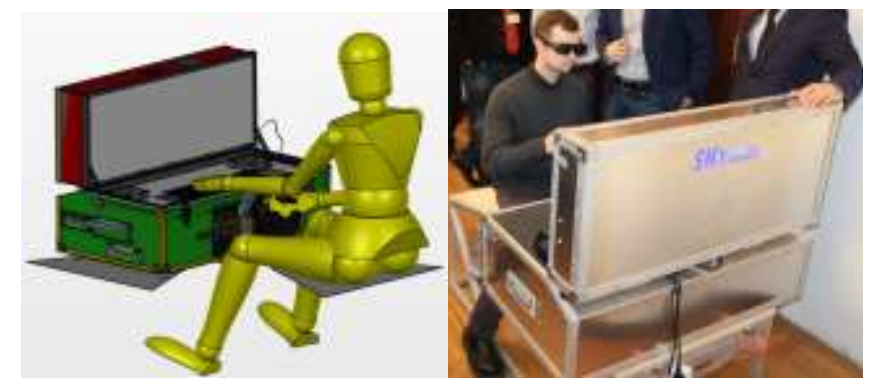

Fig. 22 Design of the operator station.

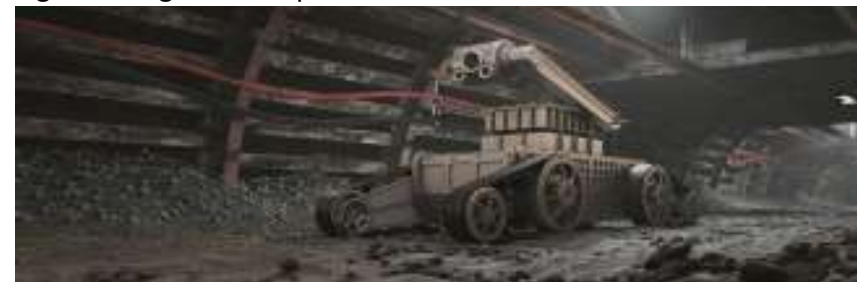

Fig. 24 Visualization of the Telerescuer robot in operational conditions. Source: [9]

\section{Iv. Conclusions}

The human-machine interface of the Telerescuer system is ready to use. However, the whole system is currently at the stage before final integration. After performing integration it will be thoroughly tested and demonstrated in nearly operational condition in a coal mine Guido (Zabrze, Poland). The demonstration is planned in the end of 2017.

\section{Acknowledgment}

This work has been partially supported by the Research Fund for Coal and Steel under the grant No. RFCR-CT-201400002, and by Polish Ministry for Science and Higher Education from financial sources that constitute the public aid in years 2014-2017 assigned for an accomplishment of an international co-financed project.

\section{References}

[1] P. Novak, J. Bajak, T. Kot, L. Olivka, and W. Moczulski "Exploration mobile robot for coal mines. In: J. Hodicky (Ed.), Modelling and simulation for autonomous systems", Second international workshop, MESAS 2015, Prague, Czech Republic, April 29-30, 2015 (Lecture Notes in Computer Science ; vol. 9055 0302-9743, pp. 209-215), Springer, 2015.

[2] L. Kasprzyczak "Mobile platform for inspection on the background of global solutions of mining robots" [in Polish], in: K. Krauze (Ed.), II Konferencja „Mechanizacja, Automatyzacja i Robotyzacja w Górnictwie, Ustroń, 2015.

[3] P. Novak, J. Babjak, and W. Moczulski "Control System of the Mobile Robot TELERESCUER", Applied Mechanics and Materials, vol. 772, pp. 466-470, 2015.

[4] I. Dryanovski, W. Morris, and X. Jizhong "Multi-volume occupancy grids: An efficient probabilistic 3D mapping model for micro aerial vehicles", Intelligent Robots and Systems (IROS), IEEE/RSJ International Conference, pp. 1553-1559, 2010.

[5] A. Hornung, K.M. Wurm, M. Bennewitz, C. Stachniss, and W. Burgard "OctoMap: an efficient probabilistic 3D mapping framework based on octrees", Autonomous Robots, vol. 34, no. 3, pp. 189-206, 2013.

[6] A.H. Watson and T.J. McCabe, "Structured Testing: A Testing Methodology Using the Cyclomatic Complexity Metric (NIST Special Publication 500-235", McCabe Software web site: http://www.mccabe.com/pdf/mccabe-nist235r.pdf, Retrieved on May 14, 2011.

[7] S.R. Chidamber and C.F. Kemerer "A Metrics Suite for Object Oriented Design”, IEEE Transactions on Software Engineering, Vol. 20, No. 6, 1994.

[8] H. Kabaili, R. Keller, F. Lustman, and G. Saint-Denis "Class Cohesion Revisited: An Empirical Study on Industrial Systems" Proceedings of the Workshop on Quantitative Approaches in Object-Oriented Software Engineering, Retrieved May 20, 2011, from Université de Montréal site: www.iro.umontreal.ca/ sahraouh/qaoose/papers/Kabaili.pdf

[9] W. Moczulski, K. Cyran, M. Januszka, P. Novak, and A. Timofiejczuk "Telerescuer - an innovative robotized system for supporting mining rescuers by inspecting roadways affected by catastrophes", 24th World Mining Congress Proceedings, Rio de Janeiro, pp. 93-104, October 2016. 